\title{
Argentine : une nouvelle loi nationale sur l'éducation
}

Mariano Palamidessi

Traducteur : Nuria Aligant

\section{(2) OpenEdition}

Journals

Édition électronique

URL : https://journals.openedition.org/ries/175

DOI : 10.4000/ries. 175

ISSN : 2261-4265

Éditeur

France Education international

\section{Édition imprimée}

Date de publication : 1 avril 2007

Pagination : 131-138

ISBN : 978-2-85420-569-5

ISSN : $1254-4590$

Référence électronique

Mariano Palamidessi, «Argentine : une nouvelle loi nationale sur l'éducation », Revue internationale d'éducation de Sèvres [En ligne], 44 | avril 2007, mis en ligne le 23 juin 2011, consulté le 08 juillet 2021. URL : http://journals.openedition.org/ries/175; DOI : https://doi.org/10.4000/ries.175

Ce document a été généré automatiquement le 8 juillet 2021

(c) Tous droits réservés 


\title{
Argentine : une nouvelle loi nationale sur l'éducation
}

\author{
Mariano Palamidessi \\ Traduction : Nuria Aligant
}

1 En mai 2006, le président argentin Néstor Kirchner a signé un décret visant l'organisation d'une consultation nationale autour de la nouvelle Loi sur l'éducation. Dans un message adressé à la nation, le Président et le ministre de l'éducation, de la science et de la technologie, Daniel Filmus, ont déclaré que «l'Argentine a besoin d'un grand débat qui contribue à l'élaboration d'un projet de loi sur l'éducation nationale légitimé par l'opinion publique et la participation de l'ensemble des citoyens, afin de construire l'Argentine que nous voulons $»^{1}$. Cette loi devait remplacer la Loi fédérale sur l'éducation de 1993 adoptée par le gouvernement de Carlos Menem.

2 Le processus qui devait conduire à la promulgation de la nouvelle loi s'est déroulé selon un calendrier très serré, comportant des phases de préparation, de consultation et de rédaction $^{2}$ : consultations auprès des institutions et des experts concernés (mai); débat général et journée de discussion dans les écoles (juin); systématisation des informations (juillet) ; avant-projet (août); deuxième phase de consultations auprès des institutions et des experts (septembre); rédaction finale et vote du projet de loi par le Congrès national (octobre).

3 Tout au long de son cheminement, l'initiative du gouvernement a bénéficié du soutien actif des principales organisations syndicales d'enseignants, des organisations non gouvernementales et des droits de l'homme ainsi que des principaux moyens de communication du pays. La proposition de réforme, relayée par une campagne de communication massive et intense, a été favorablement accueillie par l'opinion publique.

4 Cependant, certaines corporations enseignantes ont émis des réserves sur les conditions dans lesquelles s'était déroulé le débat, s'interrogeant sur la place prioritaire qu'il convenait d'accorder à la réforme de la loi ${ }^{3}$. D'autre part, divers membres de l'Église catholique ont souligné l'absence de dispositions liées à 
l'enseignement et aux orientations et contenus religieux, tandis que des associations militant pour la défense de l'éducation publique ainsi que des partis de gauche déploraient l'absence d'une définition plus claire du caractère laïque de l'éducation publique.

Enfin, le 27 décembre 2006, le président Kirchner a promulgué la Loi sur l'éducation nationale (loi $n^{\circ} 26.206$ ), après son vote par le Congrès national le 14 décembre, au cours d'une courte séance parlementaire. Le parti officiel (Parti justicialiste - Front pour la victoire), qui disposait de la majorité dans les deux instances législatives, a réussi ainsi une « adoption rapide » de la loi, quasiment sans ajustement.

Lors de la présentation aux médias de l'entrée en vigueur de la nouvelle loi, le ministre Filmus était accompagné de Hugo Yasky et de Francisco Nenna, respectivement représentants de la Centrale des travailleurs de l'éducation de la République argentine (CTERA) et de l'Union des travailleurs de l'éducation de la ville de Buenos Aires (UTE), ce qui traduit le soutien dont bénéficiait le projet de loi de la part des principaux syndicats enseignants du pays. Dans son allocution, le ministre a déclaré que la loi était «le résultat d'un débat participatif et fécond auquel ont participé tous les acteurs du système éducatif» tandis que le secrétaire général de la CTERA qualifiait la promulgation de la Loi sur l'éducation nationale de « fait historique ».

7 Dans les jours qui ont suivi, les journaux les plus influents du pays ont fait leurs titres autour des principaux axes de la nouvelle loi : allongement de la scolarité obligatoire (de dix à treize ans) et retour à l'organisation par niveaux et cycles du système éducatif antérieure à la réforme éducative mise en œuvre dans les années $1990^{4}$. Pour leur part, les syndicats enseignants ont salué l'abrogation de la Loi fédérale sur l'éducation 5 .

\section{L'abrogation de la Loi fédérale sur l'éducation}

8 Quelle est la raison qui a conduit le gouvernement argentin à remplacer la Loi fédérale sur l'éducation de 1993 par la nouvelle Loi sur l'éducation nationale?

9 La Loi fédérale sur l'éducation de 1993 a été la première loi à réguler l'éducation en Argentine, dans l'ensemble de ses domaines et de ses modalités. Elle réglementait une politique fédéraliste articulée autour d'un ensemble de responsabilités partagées et complémentaires ente l'État et les provinces. Par ailleurs, elle allongeait de trois ans la scolarité obligatoire (de sept à dix ans), établissait un changement substantiel concernant l'organisation des niveaux, cycles et modalités d'enseignement et réformait les contenus fondamentaux de l'ensemble du système y compris celui de la formation des enseignants.

10 En définitive, elle proposait une réforme des dispositifs existant depuis la fin du XIX siècle, basés sur une école primaire obligatoire d'une durée de sept ans pour les enfants âgés de six à douze ans ainsi que d'un enseignement secondaire d'une durée de cinq ou six ans (selon les séries et les modalités), pour les jeunes âgés de douze à dix-huit ans ${ }^{6}$.

11 Bien qu'elle ait suscité l'opposition d'une partie des syndicats enseignants et de certains secteurs sociaux - académiques et intellectuels ainsi que des partis de gauche et des groupes pour la défense de l'éducation publique - , la Loi fédérale sur l'éducation disposait, en réalité, d'un solide soutien. Son adoption a bénéficié de l'appui sans réserve du Parti justicialiste et de la quasi-totalité des députés de l'opposition et la mise en œuvre du processus de réforme éducative a été plébiscité par une large frange de 
l'opinion publique ainsi que par les enseignants qui y ont vu la réponse à une réelle nécessité.

Cependant, le contexte économique, politique et institutionnel de l'époque était particulièrement complexe : un projet de décentralisation de la politique éducative décidé par le gouvernement de façon unilatérale et sans consultation préalable avait précédé l'entrée en vigueur de la loi et des réformes qu'elle préconisait. Il portait sur le transfert de compétences de gestion des établissements scolaires de l'État vers les provinces: les écoles pendant la dictature militaire (1979) et les établissements du second degré pendant les premières années du gouvernement Menem (1991). C'est ainsi que, lors de l'entrée en vigueur de la Loi fédérale, les provinces et la ville autonome de Buenos Aires se sont trouvées confrontées à des problèmes majeurs concernant la mise en œuvre d'une réforme générale à grande échelle, alors qu'elles devaient réguler et gérer leurs systèmes éducatifs dont la taille et la complexité s'étaient accrues de façon abrupte ${ }^{7}$.

Par ailleurs, en 1994-1995, la réforme éducative avait été mise en œuvre sans qu'un diagnostic préalable soit posé qui éclaire sur les conditions réelles de fonctionnement du système éducatif et sur les ressources financières et humaines dont disposaient les provinces à ce moment-là. Le contexte était très incertain: désordre normatif et administratif, faible niveau d'informatisation, personnels peu professionnalisés. C'est ainsi que chaque province a dû improviser des stratégies propres de mise en œuvre, ce qui a provoqué un accroissement de l'anomie institutionnelle, de l'inégalité en matière de financements et de ressources affectés, ainsi que la prolifération chaotique d'ajustements institutionnels ${ }^{8}$.

Lors de la mise en application de cette politique de décentralisation générale et accélérée, la capacité de coordination politique et technique du système éducatif s'est affaiblie tandis que la crise financière exacerbait les conflits relatifs au versement des traitements salariaux dans la quasi-totalité de provinces du pays. À mesure que progressait la mise en œuvre de la loi, les syndicats d'enseignants - majoritairement représentés par la CTERA - sont passés d'une position attentiste à une position clairement critique et combative qui a débouché sur la demande d'abrogation de la Loi fédérale sur l'éducation.

15 À la fin des années quatre-vingt-dix, la réforme éducative avait perdu de son élan initial et, en 2000-2001, elle se trouvait dans une impasse. D'autre part, elle ne disposait plus de la légitimité sociale qui l'avait portée à ses débuts. La fédéralisation du système éducatif n'a pas résolu les problèmes dus à la centralisation et le bilan social de l'expérience a été jugé de façon très négative ${ }^{9}$. Vers la fin du deuxième mandat de Carlos Menem, la Loi fédérale sur l'éducation a été assimilée aux politiques néolibérales de privatisation du secteur public et d'ouverture économique qui ont débouché sur la crise économique et sociale qui a éclaté dans les années 2001-2002, entraînant une chute générale de l'activité économique et un accroissement sans précédent de la pauvreté, du chômage et de l'exclusion.

16 Cela explique la raison pour laquelle, au cours de ces dernières années, la consigne visant à abroger la Loi fédérale sur l'éducation ${ }^{10}$, fortement relayée par les syndicats d'enseignants, d'étudiants et les partis de gauche, a gagné du terrain auprès des différents secteurs sociaux et politiques du pays.

À partir de 2003, le président Kirchner, porté par une embellie économique qui l'a aidé à se forger une image indéniablement positive auprès de la société argentine, a décidé 
de promouvoir la mise en œuvre d'une série de lois relatives à l'éducation qui visaient à remplacer les lois en vigueur depuis la décennie précédente ${ }^{11}$, ce qui a eu pour effet de "débloquer» la situation de paralysie dans laquelle se trouvait l'éducation. L'avantprojet de loi générale proposé par l'exécutif a été incontestablement soutenu par une ample frange de l'opinion qui y a vu l'opportunité de rétablir la division traditionnelle entre premier et second degrés, de redonner à l'État son rôle de régulateur et de garant du droit à l'éducation, d'octroyer une plus grande unité et cohérence au système éducatif et, en même temps, de proposer d'autres objectifs de scolarité et d'apprentissage pour les enfants et les jeunes.

\section{Principaux contenus et orientations de la nouvelle loi}

La Loi sur l'éducation nationale est une loi large et détaillée qui s'inscrit dans la tradition argentine : elle est composée de huit chapitres et 145 articles. En opposition discursive avec les orientations politiques et culturelles dominantes dans les années quatre-vingtdix, elle comporte un important dispositif de droits et de garanties individuels et sociaux et resitue l'État au centre du système éducatif. Elle établit entre autres, les dispositions suivantes :

- l'éducation est un bien public et un droit personnel et social garanti par l'État national (article 2);

- l'État assume seul sa principale responsabilité : «garantir à tous les citoyens l'accès à une éducation intégrale, continue et de qualité ainsi que l'égalité, la gratuité et l'équité de chacun dans l'exercice de ce droit, avec le concours des organisations sociales et parentales » (article 3);

- concernant les objectifs de la politique éducative nationale, la loi souligne que l'éducation est une "priorité nationale et une politique d'État qui contribue à construire une société juste, à réaffirmer la souveraineté et l'identité nationales ainsi qu'à consolider l'exercice de la citoyenneté et le développement économique et social de la nation » (article 4);

- l'État garantit la dotation du système éducatif national en ressources financières et déclare que «le budget de l'État national, des provinces et de la ville autonome de Buenos Aires consacré à l'éducation ne pourra être inférieur à $6 \%$ du PIB » (article 9).

Quant au chapitre relatif à l'allongement de la scolarité obligatoire et à l'organisation des différents niveaux et cycles du système éducatif, il prévoit :

Concernant l'enseignement secondaire, la loi préconise :

Concernant les groupes, secteurs et personnes exclus et/ou marginalisés du système éducatif, la loi prévoit le développement de "politiques de promotion de l'égalité éducative, destinées à combattre les situations d'injustice, de marginalisation, de stigmatisation ainsi que toutes autres expressions de discrimination, dérivées de facteurs socio-économiques, culturels, géographiques, ethniques, de genre ou de toute autre nature, susceptibles d'entraver l'exercice du plein droit à l'éducation (article 79). Concernant les enseignants («facteur clé de l'amélioration de la qualité de l'éducation »), la loi envisage :

Quant aux contenus communs des programmes d'enseignement :

Pour ce qui est de l'articulation politique et de la gestion du système éducatif : 


\section{Défis nouveaux, problèmes récurrents}

L'adoption de la Loi sur l'éducation nationale ouvre une opportunité nouvelle pour réglementer, améliorer et développer l'éducation argentine dans les premières décennies $\mathrm{du} \mathrm{XXI}{ }^{e}$ siècle. Elle définit des objectifs fondés sur la concertation et la légitimité ; elle reconnaît un ensemble de droits et de garanties et renforce et relance les valeurs et symboles qui ont forgé la meilleure tradition de l'éducation publique argentine; elle prévoit une réorganisation institutionnelle cohérente qui permette d'atteindre les objectifs d'élargissement et d'amélioration de l'éducation.

La nouvelle loi définit un programme très audacieux et un agenda de travail compliqué à mettre en place puisqu'il devra combiner processus de généralisation et de démocratisation de l'éducation à grande échelle, projets de modernisation et de réorganisation institutionnelle et amélioration des pratiques pédagogiques. Compte tenu du caractère à la fois complexe et très ambitieux des objectifs définis et des difficultés certaines à les atteindre à court et moyen terme, la mise en œuvre peut s'avérer délicate. Par conséquent, pendant l'année 2007, le Conseil fédéral de l'éducation, qui réunit les ministres des vingt-trois provinces et de la ville autonome de Buenos Aires, devra définir le calendrier portant sur «l'harmonisation au niveau national de la structure du système éducatif » prévu à l'article 133 ; « la journée scolaire élargie ou complète pour le premier degré " (article 134) et la "réforme de l'enseignement secondaire " (article 135); la planification des programmes d'enseignement, des activités et des actions d'accompagnement afin d'atteindre les objectifs fixés par la loi, avec leurs objectifs, calendriers de réalisation et ressources affectés ; et, enfin, les dispositifs de suivi et d'évaluation prévus.

Le défi pour les prochaines années consiste à définir les meilleurs moyens de parvenir à formaliser des accords progressifs qui s'inscrivent dans le temps, et à consolider le consensus social qui a fondé la légitimité du texte de loi afin qu'il puisse trouver sa traduction au moyen de projets concrets, systématiquement évalués, et ce avec le concours des différents acteurs sociaux et institutionnels.

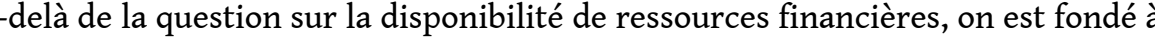
s'interroger sur l'indéniable faiblesse des dispositifs institutionnels, politiques et techniques qui doivent accompagner et garantir le succès d'un projet de développement éducatif d'une telle nature et d'une telle complexité. Le Conseil fédéral est un organisme consultatif dont l'efficacité du fonctionnement reste encore à prouver. D'autre part, et d'un point de vue global, les niveaux de planification et de coordination des politiques fédérales sont extrêmement précaires et discontinus.

Outre les fortes inégalités économiques qui frappent la société argentine en général, il en existe qui agissent directement sur la répartition des ressources - financières, institutionnelles et techniques - au niveau des provinces. Une grande partie de la bureaucratie éducative se caractérise par sa faible capacité à gérer efficacement le système et à agir sur l'optimisation des données ainsi que sur la qualité de l'enseignement dans les établissements scolaires. Par conséquent, la mise en place d'une articulation institutionnelle plus efficace s'avère impérative pour atteindre les droits et les garanties souhaités et parvenir aux objectifs définis.

30 La nouvelle Loi nationale sur l'éducation parviendra-t-elle à dépasser le cycle espoir/ désenchantement qui a caractérisé la vie politique et institutionnelle argentine de ces 
dernières décennies? Réussira-t-elle à secouer l'inertie et le conformisme qui imprègnent une frange non négligeable de la culture et du comportement des institutions éducatives?

31 Malgré le contexte de croissance économique et l'image très positive dont bénéficie le gouvernement actuel, force est de constater que le consensus politique et institutionnel peut être extrêmement fluctuant en Argentine. Malgré l'optimisme légitime et porteur déclenché par l'adoption et la mise en œuvre de la Loi nationale sur l'éducation, la récente expérience de la Loi fédérale sur l'éducation devrait agir comme un rappel à la vigilance et à la prudence.

Il serait souhaitable que les acteurs politiques, les administrations scolaires, les organisations de la société civile, les enseignants, les académiques ainsi que les parents d'élèves retiennent les leçons de l'histoire pour faire éclore et articuler les volontés et les capacités dont ils ont besoin pour entreprendre avec succès les missions que propose la nouvelle Loi.

\section{BIBLIOGRAPHIE}

CARLI S. Apuntes críticos sobre el documento base: Ley de educación nacional. Hacia una educación de calidad para una sociedad más justa, en Jornadas de Reflexión Pública « La educación en debate: Desafíos para una nueva Ley », Escuela de Ciencias de la Educación, Universidad Nacional de Córdoba, agosto de 2006, 2006.

Confederación de trabajadores de la educación de la República Argentina. « Se derogó la Ley Federal de Educación », comunicado de prensa de la Secretaria Prensa y Cultura, Buenos Aires, 15 de diciembre, 2006.

Departamento de ciencias de la educación de la facultad de filosofía y letras. Declaración sobre el debate de la nueva Ley de Educación Nacional, Facultad de Filosofía y Letras, Universidad de Buenos Aires, 2006.

CLARIN D. Editorial. La educación, pilar del progreso y la equidad, Buenos Aires, 3 de enero, 2007.

HIRSCHBERG S. Implementación y localización del Tercer Ciclo de la EGB. Las prescripciones y su impacto en los actores institucionales, Ministerio de Educación, Buenos Aires, 2000.

Ministerio de educación, ciencia y tecnología. Ley de Educación Nacional. Hacia una educación de calidad para una sociedad más justa. Documento base. Buenos Aires, 2006.

Poder ejecutivo nacional. Ley de Educación Nacional. № 26.206 (disponible en http:// www.me.gov.ar/prensa/gacetilla/docs/ley_nacional.pdf).

Presidencia de la nación. «Convocatoria a un debate abierto y profundo », Mensaje del Presidente de la Nación y el Ministro de Educación, Ciencia y Tecnología, Buenos Aires, 22 de mayo de 2006. 


\section{NOTES}

1. Présidence de la Nation, 22 mai 2006.

2. «Le pouvoir exécutif national a considéré également le besoin d'établir un cadre défini de cent quatre-vingts jours pour conduire les consultations, débats et études liés à la préparation de la Loi sur l'éducation nationale » (idem).

3. Entre autres, voir Carli (2006) et Département en sciences de l'éducation de la faculté de philosophie et lettres (2006).

4. L'agence $D Y N$ a signalé : « Le président Néstor Kirchner a promulgué ce matin la nouvelle Loi nationale sur l'éducation qui établit une période d'enseignement obligatoire de treize ans, parmi d'autres changements » (27 décembre 2006). Le même jour, le journal Clarín annonçait « le retour de l'enseignement secondaire ». Le journal Perfil a souligné : " avec la nouvelle Loi sur l'éducation nationale et à partir de l'année prochaine, l'enseignement secondaire sera également obligatoire ».

5. Dans un communiqué intitulé «Abrogation de la Loi fédérale sur l'éducation », la CTERA (2006) a souligné que "le projet approuvé hier comporte de nombreux points débattus lors de l'Assemblée nationale des organisations sociales par les enseignants et les organisations des droits de l'homme, qui avaient été soumis au gouvernement ". Parmi ces questions, on trouve : l'éducation doit s'entendre comme un "bien public et un droit social »; la volonté de " réunification du système éducatif "; le soutien au " rôle primordial de l'État en tant que garant du financement et de l'organisation des enseignements dans le premier et le second degré » et l'organisation du volet concernant la formation des maîtres.

6. La Loi fédérale était articulée comme suit : a) educación inicial (correspondant à l'école maternelle) pour les enfants âgés de trois à cinq ans, dont la dernière année obligatoire ; b) educación general básica (correspondant à l'école élémentaire et aux deux premières années de collège), obligatoire, d'une durée de neuf ans ; c) educación polimodal (correspondant au lycée) pendant trois ans ; d) educación superior (enseignements supérieur) ; enseignement technique et professionnel ; autres enseignements (enseignement spécial, d'adultes et artistique).

7. Voir Insès Aguerrondo : "Centraliser pour décentraliser : le renforcement du ministère national pour développer l'organisation fédérale en Argentine ", Revue internationale d'éducation de Sèvres $n^{\circ}$ 32, mars 2003, (Le processus de décision dans les systèmes éducatifs), pp. 29-41.

8. Ministère de l'éducation, 2000.

9. Ce bilan a fait l'objet d'un récent éditorial publié par le journal Clarín : « la décentralisation des établissements scolaires vers les collectivités territoriales n'a pas bénéficié des mesures d'accompagnement budgétaires indispensables ni de la nécessaire harmonisation des contenus des programmes d'enseignement (...). L'éducation publique dans les zones les plus défavorisées du pays a perdu son potentiel de transformation pour devenir un service éducatif également paupérisé. La disparité dans les contenus des enseignements dispensés dans chaque juridiction ne répond à aucun critère raisonnable ni fédéral. Par ailleurs, les retards dans le versement des salaires aux enseignants n'ont fait qu'exacerber le conflit, aggravant la déroute institutionnelle de l'école publique » (3 janvier 2006).

10. "Après des années de résistance et de lutte de la part des enseignants, des étudiants et des parents d'élèves, la Loi Fédérale sur l'éducation, source de fragmentation et d'atomisation du système public d'enseignement a été abrogé », CTERA, décembre 2006.

11. Avant d'envoyer au congrès la Loi sur l'éducation nationale, le gouvernement avait déjà réussi l'approbation de la Loi sur l'enseignement technique et professionnel (loi 26.058, septembre 2005) ainsi que de la Loi sur le financement éducatif (Loi 26.075, janvier 2006). 
INDEX

Mots-clés : politique éducative, réforme de l'enseignement, système éducatif Index géographique : Argentine

\section{AUTEURS}

MARIANO PALAMIDESSI

Professeur, Escuela de Educación de la Universidad de San Andrés, Argentine. 